\title{
Prognostic Effect of the Dose of Loop Diuretic Over 5 Years in Chronic Heart Failure
}

\author{
OLGA LASZCZYŃSKA, MSc, ${ }^{1}$ MILTON SEVERO, PhD, ${ }^{1,2}$ FERNANDO FRIÕES, MD, PhD,, 3 PATRÍCIA LOURENÇO, MD, PhD, ${ }^{3,4}$ \\ SÉRGIO SILVA, MD,${ }^{3}$ PAULO BETTENCOURT, MD, PhD,${ }^{3,4}$ NUNO LUNET, PharmD, MPH, PhD, ${ }^{1,2}$ AND \\ ANA AZEVEDO, MD, $\mathrm{PhD}^{1,2}$ \\ Porto, Portugal
}

\begin{abstract}
Background: High diuretic doses in chronic heart failure (HF) are potentially deleterious. We assessed the effect of dynamic furosemide dose on all-cause mortality among HF ambulatory patients.

Methods and Results: A cohort of 560 ambulatory patients from an outpatient clinic specialized in HF, with median age 70 years, $67 \%$ male, and $89 \%$ with moderate-severely reduced ejection fraction, was retrospectively followed for up to 5 years. Dynamic furosamide exposure was categorized as low (0-59 mg/ d), medium (60-119 mg/d), high (120-159 mg/d), and very high ( $\geq 160 \mathrm{mg} / \mathrm{d})$. Extended Cox models were used to estimate the association between time-varying diuretic dose and mortality. A dose-dependent crude association between higher doses of furosemide and death (hazard ratio $[\mathrm{HR}]=1.34,95 \%$ confidence interval (CI): 1.06-2.16; HR $=2.09,95 \% \mathrm{CI}: 1.54-2.84$, for high and very high dose, respectively) was totally explained by patients' characteristics and disease severity indicators (adjusted HR $=0.94,95 \%$ CI: 0.63 $1.38 ; \mathrm{HR}=1.10,95 \% \mathrm{CI}: 0.79-1.55$, for high and very high dose, respectively).

Conclusion: In this context, higher doses of diuretic did not impair survival, but rather indicated greater severity of the patient's condition. (J Cardiac Fail 2017;23:589-593)

Key Words: Heart failure, loop diuretics, dose, prognosis.
\end{abstract}

Congestion is a key component of heart failure (HF) that determines symptoms, hospital admissions, and mortality. ${ }^{1}$ Of all drugs used in HF, diuretics are the most efficacious for relief from fluid congestion, ${ }^{2}$ thus becoming the most frequently prescribed drug class ${ }^{3}$ despite the limited evidence to guide their prescription and titration. The use of inappropriately low doses of diuretics may lead to persistent edema,

From the ${ }^{1}$ EPIUnit-Instituto de Saúde Pública, Universidade do Porto, Rua das Taipas $n^{\circ}$ 135, 4050-600 Porto, Portugal; ${ }^{2}$ Departamento de Ciências da Saúde Pública e Forenses e Educação Médica, Faculdade de Medicina da Universidade do Porto, Alameda Prof. Hernâni Monteiro, 4200-319 Porto, Portugal; ${ }^{3}$ Heart Failure Clinic, Department of Internal Medicine, Centro Hospitalar de São João, Alameda Prof. Hernâni Monteiro, 4202-451 Porto, Portugal and ${ }^{4}$ Cardiovascular Research Centre, University of Porto Medical School, Alameda Prof. Hernâni Monteiro, 4200-319 Porto, Portugal.

Manuscript received August 11, 2016; revised manuscript received March 28, 2017; revised manuscript accepted April 4, 2017.

Reprint requests: Olga Laszczyńska, M.Sc., EPIUnit-Instituto de Saúde Pública, Universidade do Porto, Rua das Taipas n ${ }^{\circ} 135$, 4050-600 Porto, Portugal. Tel: +351 222061 820; Fax: +351 222061 821. E-mail: olaszczynska@med.up.pt.

See page 593 for disclosure information.

1071-9164/\$ - see front matter

(c) 2017 Elsevier Inc. All rights reserved.

http://dx.doi.org/10.1016/j.cardfail.2017.04.001 whereas high doses may result in volume contraction, which can increase the risk of hypotension and renal insufficiency. ${ }^{4}$

According to guidelines, the aim of using diuretics is to achieve and maintain a euvolemic state and patients' wellbeing with the lowest achievable dose. ${ }^{5}$ This requires individual diuretic dose adjustments given the progressive character of $\mathrm{HF}$, its concomitant conditions and treatments, and changes in dietary sodium and fluid intake over time. In this study, we assess the association of the fluctuating dose of furosemide with all-cause mortality over 5 years in ambulatory patients within a wide spectrum of HF presentation.

\section{Methods}

For this retrospective cohort study, we screened 765 consecutive ambulatory patients referred between January 2000 and July 2011 to the HF clinic at Hospital São João in Porto, Portugal. Inclusion criteria of HF diagnosis ${ }^{5}$ and reduced ejection fraction $(\leq 50 \%)$ were confirmed in 632 patients; those with uncorrected primary valvular disease $(\mathrm{n}=10)$, on dialysis $(n=4)$, and attending only 1 appointment at the clinic $(\mathrm{n}=58)$ were excluded, leaving 560 patients for analysis. 
Data on patients' characteristics, prescribed therapy, and laboratory parameters were abstracted from records of each clinical appointment during the study period, corresponding to 4978 observations. Dynamic diuretic exposure was determined based on furosemide dose prescribed at each medical visit. After consultation with cardiologists specialized in HF, the daily dose was categorized as low (0$59 \mathrm{mg}$ ), medium (60-119 mg), high (120-159 mg), and very high doses ( $\geq 160 \mathrm{mg})$. Qualitative assessment of left ventricular ejection fraction was considered as documented in clinical records. $\mathrm{N}$-terminal prohormone of brain natriuretic peptide (BNP) values were converted into $\mathrm{BNP}^{6}$ and serum sodium $\leq 133 \mathrm{mEq} / \mathrm{L}$ was defined as moderate or severe hyponatremia.?

Patients were followed until July 31, 2012, for the endpoint of all-cause mortality. Vital status was ascertained using the clinic's records and telephone contacts. Patients were censored if regular hemodialysis was instituted, there was no appointment at the clinic for more than 15 months, or after 5 years after referral, whichever came first. The study was approved by the institutional ethics committee.

\section{Statistical Analysis}

Baseline dose categories were compared using the $\chi 2$ test for discrete and 1-way analysis of variance or KruskalWallis tests for continuous variables. Survival was estimated using the Kaplan-Meier method. Forty-five percent of observations had no data on at least 1 laboratory parameter, to a large extent representing no need for tests in stable patients. Data on variables other than laboratory parameters were lacking in $6 \%$ of observations. Absent and missing data, as well as nonexistent $\mathrm{BNP}$ values (N-terminal prohormone of BNP/BNP blood test became available at the hospital after 2003) were handled by multiple imputation using mixed effects multivariable regression models. Extended Cox models were used to assess the association between dynamic diuretic dose and all-cause mortality. The dynamic dose was sequentially adjusted for the baseline dose (Table 1, model 2), timeindependent confounders (age, sex, ischemic etiology, diabetes mellitus, and atrial fibrillation) (model 3), time-varying disease severity indicators (hospital admissions resulting from HF,
New York Heart Association [NYHA] class, and BNP) (model 4), time-varying laboratory and physiologic parameters (creatinine, sodium, and systolic blood pressure), (model 5) and concomitant disease-modifying treatments (angiotensinconverting enzyme inhibitors, angiotensin II receptor blockers, and $\beta$-blockers) (model 6).

Sensitivity analyses were performed to examine the effect of increased dose of furosemide on 2-year survival. Statistical analyses were performed using Stata and R softwares. $P$ values $<.05$ were considered significant.

\section{Results}

Overall characteristics at referral included median age of 70 years, $67 \%$ male, $46 \%$ of ischemic etiology, $89 \%$ with moderateseverely reduced EF, and 62\% in NYHA class II (Table 2).

Higher referral daily diuretic doses were related with clinical markers of more severe disease, including higher NYHA class, higher BNP and serum creatinine, lower systolic blood pressure, and lower hemoglobin levels. Patients on higher daily diuretic doses were more often hospitalized for HF in the preceding year and more frequently had comorbidities. Over 6 months and 5 years of follow-up, $85 \%$ and $40 \%$ of patients remained in the same diuretic dose category as at baseline, respectively (Fig. 1).There were 109 deaths during the followup period, with increasing 5 -year risk of death across referral diuretic dose categories $(26 \%, 32 \%, 43 \%$, and $56 \%$ in low, medium, high, and very high dose groups, respectively; logrank test: $P<.001)$.

In univariable analysis of dynamic dose (Table 1, model 1) a higher furosemide dose was associated with a progressively higher mortality. When adjusted for baseline dose (model 2) and other time-independent confounders (model 3), very high dose was independently related with higher risk of death; however, this association became close to null after controlling for HF severity (model 4). Subsequent adjustment for serum creatinine, systolic blood pressure, and hyponatremia (model 5), and additionally for prognosis-modifying therapy (model 6), had no impact on the association between diuretic dose and risk of death.

In sensitivity analysis, high furosemide dose prescribed at referral was associated with higher 2-year mortality, whereas

Table 1. Hazard ratio for mortality according to time-varying diuretic dose categories over 5 years, adjusted for the baseline dose and multiple confounders

\begin{tabular}{|c|c|c|c|c|c|}
\hline Model & Low & Medium & High & Very High & Variables Included \\
\hline 1 & $1 *$ & $1.38(1.00-1.89)$ & $1.51(1.06-2.16)^{\dagger}$ & $2.09(1.54-2.84)^{\dagger}$ & Time-varying diuretic dose \\
\hline 2 & $1 *$ & $1.28(0.93-1.77)$ & $1.34(0.93-1.93)$ & $1.85(1.36-2.53)^{\dagger}$ & Baseline diuretic dose + time-varying diuretic dose \\
\hline 3 & $1 *$ & $1.18(0.86-1.61)$ & $1.22(0.85-1.76)$ & $1.63(1.19-2.22)^{\dagger}$ & $\begin{array}{l}\text { Model } 2+\text { age }+ \text { sex }+ \text { ischemic etiology }+ \text { atrial fibrillation }+ \text { diabetes } \\
\text { mellitus (baseline) }\end{array}$ \\
\hline 4 & $1^{*}$ & $1.06(0.76-1.45)$ & $0.94(0.65-1.37)$ & $1.06(0.76-1.47)$ & $\begin{array}{l}\text { Model } 3+\text { NYHA class + BNP + HF hospitalization (baseline and } \\
\text { time-varying) }\end{array}$ \\
\hline 5 & $1^{*}$ & $1.08(0.78-1.49)$ & $0.97(0.66-1.43)$ & $1.12(0.80-1.57)$ & $\begin{array}{l}\text { Model } 4+\text { serum creatinine }+ \text { blood pressure }+ \text { hyponatremia (baseline and } \\
\text { time-varying) }\end{array}$ \\
\hline 6 & $1 *$ & $1.11(0.80-1.54)$ & $0.94(0.63-1.38)$ & $1.10(0.79-1.55)$ & Model $5+$ ACE inhibitor $+\beta$-blocker treatment (baseline and time-varying) \\
\hline
\end{tabular}

$\mathrm{N}=560$ patients; 4978 observations; absent data in each observation (missing or no laboratory tests ordered) were imputed

*Reference class.

${ }^{\dagger} P<.05$ vs. low dose. 
Table 2. Patient Characteristics at Baseline

\begin{tabular}{|c|c|c|c|c|c|c|c|}
\hline & & All & Low Dose & Medium Dose & High Dose & Very High Dose & Pvalue \\
\hline No. of patients & & 560 & 164 & 252 & 93 & 51 & \\
\hline Age (y), median (IQR) & & $70.0(57.3-78.7)$ & $65.8(53.7-76.5)$ & $70.1(57.1-79.2)$ & $74.1(63.5-79.7)$ & $70.2(61.5-77.0)$ & .015 \\
\hline Male sex, \% & & 67.0 & 69.5 & 64.7 & 68.8 & 66.7 & .748 \\
\hline Ischemic etiology, \% & & 46.1 & 45.1 & 46.8 & 40.9 & 54.9 & .434 \\
\hline Diabetes mellitus, \% & & 35.5 & 32.3 & 34.1 & 37.6 & 49.0 & .159 \\
\hline Hypertension, $\%$ & & 61.6 & 58.5 & 62.7 & 61.3 & 66.8 & .720 \\
\hline Arial fibrillation, \% & & 37.7 & 24.4 & 40.9 & 48.4 & 45.1 & $<.001$ \\
\hline Chronic kidney disease, $\%$ & & 45.7 & 32.9 & 48.0 & 46.2 & 74.5 & $<.001$ \\
\hline Hospitalization for HF in previous year, \% & & 45.5 & 29.3 & 50.0 & 52.7 & 62.6 & $<.001$ \\
\hline Ejection fraction $(\%)$, median (IQR)* & & $26(20-35)$ & $28(21-35)$ & $27(20-35)$ & $25(21-33)$ & $25(20-30)$ & .304 \\
\hline Moderate-severe reduced EF, \% & & 88.9 & 88.4 & 88.5 & 88.2 & 94.1 & .673 \\
\hline \multirow[t]{4}{*}{ NYHA class, $\%$} & I & 18.7 & 29.2 & 18.5 & 8.0 & 6.0 & \\
\hline & II & 62.0 & 57.8 & 65.4 & 59.8 & 62.0 & \\
\hline & III & 18.0 & 13.0 & 15.2 & 28.7 & 28.0 & \\
\hline & IV & 1.3 & 0.0 & 0.9 & 3.5 & 4.9 & $<.001$ \\
\hline Systolic blood pressure (mmHg), mean (SD) & & $120.4(24.3)$ & $124.1(24.6)$ & $119.9(24.7)$ & $120.7(23.8)$ & $110.8(20.1)$ & .012 \\
\hline Heart rate (beats/min), mean $(\mathrm{SD})$ & & $78.3(13.9)$ & $78.5(12.5)$ & $78.1(14.5)$ & $78.6(14.1)$ & $78.1(15.7)$ & .985 \\
\hline $\mathrm{BNP}(\mathrm{pg} / \mathrm{mL})$, median (IQR) & & $458.5(224.8-1047.0)$ & $193.4(93.8-454.2)$ & $530.5(263.1-995.8)$ & $569.1(306.7-1441.4)$ & $1281.0(508.1-2918.5)$ & $<.001$ \\
\hline Creatinine $(\mathrm{mg} / \mathrm{dL})$, median $(\mathrm{IQR})$ & & $1.1(0.9-1.4)$ & $1.0(0.9-1.3)$ & $1.1(1.0-1.4)$ & $1.1(0.9-1.5)$ & $1.4(1.0-2.0)$ & $<.001$ \\
\hline Uric acid (mg/L), median (IQR) & & $74.9(62.7-89.2)$ & $67.0(56.4-79.2)$ & $76.6(64.8-90.8)$ & $77.7(63.4-93.9)$ & $88.4(70.3-110.5)$ & $<.001$ \\
\hline Hyponatremia, \% & & 6.7 & 6.2 & 5.2 & 6.2 & 15.7 & .057 \\
\hline Hemoglobin $(\mathrm{g} / \mathrm{dL})$, mean $(\mathrm{SD})$ & & $13.2(1.9)$ & $13.5(1.9)$ & $13.2(1.9)$ & $12.8(1.9)$ & $12.5(2.0)$ & .009 \\
\hline Diuretic dose (mg/day), median (IQR) & & $80(40-120)$ & $40(20-40)$ & $80(80-80)$ & $120(120-120)$ & $160(160-160)$ & $<.001$ \\
\hline Metolazone, $\%$ & & 2.3 & 0.0 & 1.2 & 4.3 & 11.8 & $<.001$ \\
\hline ACE inhibitors, \% & & 89.8 & 92.1 & 88.9 & 89.3 & 88.2 & .724 \\
\hline$\beta$-blocker, $\%^{\dagger}$ & & 75.4 & 74.4 & 78.6 & 65.6 & 80.4 & .073 \\
\hline Spironolactone, \% & & 32.5 & 19.5 & 36.1 & 41.0 & 41.2 & $<.001$ \\
\hline Follow-up (months), median (IQR) & & $23(9-44)$ & $26(10-48)$ & $22(10-41)$ & $19(8-40)$ & $16(6-51)$ & .192 \\
\hline Time between appointments (days), median (IQR) & & $79(41-123)$ & $94(54-153)$ & $83(41-118)$ & $62(34-104)$ & $62(34-97)$ & $<.001$ \\
\hline No of visits/patient & & $8(4-12)$ & $8(4-11)$ & $8(4-13)$ & $7(3-13)$ & $8(3-15)$ & .848 \\
\hline
\end{tabular}

ACE, angiotensin-converting enzyme; BNP, brain natriuretic peptide; COPD, chronic obstructive pulmonary disease; EF, ejection fraction; HF, heart failure; IQR, interquartile range; MI, myocardial infarction; NYHA, New York Heart Association; SD, standard deviation.

*Data available for 264 patients.

Carvedilol, bisoprolol, or nebivolol. 

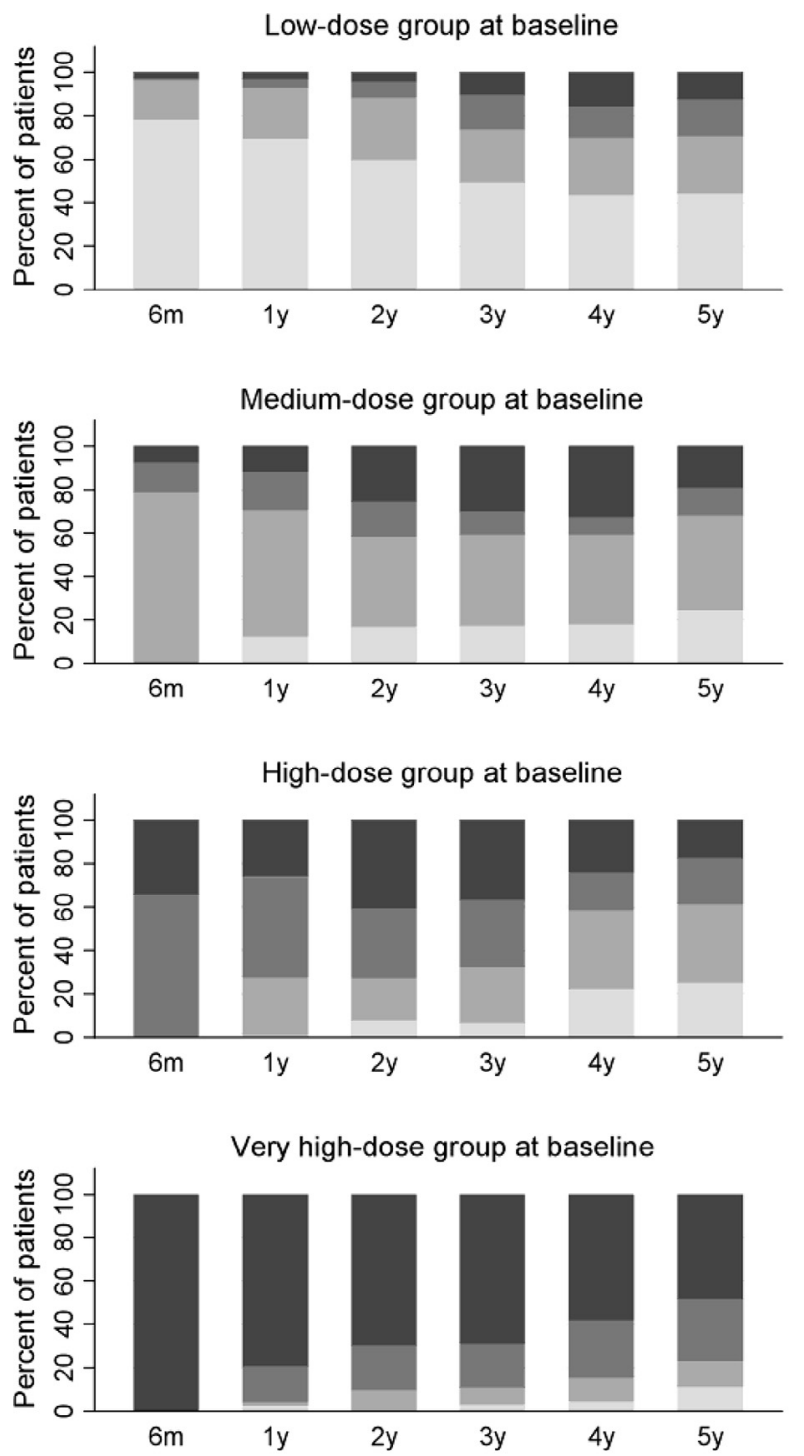

low dose medium dose high dose very high dose

Fig. 1. Diuretic dose categories distribution over 5 years according to furosemide dose at baseline.

time-varying dose showed no effect on risk of death Appendix S1, (Supplementary Table A, model 6).

\section{Discussion}

Higher doses of furosemide were strongly associated with subsequent death, with a clear dose-dependent increase in crude risk. However, the association was totally explained by patients' characteristics and confounders reflecting the severity of the patient's condition and greater comorbidities.

Our conclusion that diuretics dose may be more a marker than a cause of instability is consistent with previous reports. ${ }^{3,8}$ At the same time, high diuretic doses were associated with increased rehospitalizations and mortality. ${ }^{9}$ Evaluation of the prognostic effect of diuretic in chronic therapy based on single baseline dose only may be a simplification, especially for long- term perspective. Currently, there is no specific strategy for diuretic treatment in $\mathrm{HF}$, and recommendations are for the minimum dose to allow maintenance of the euvolemic state. ${ }^{5}$ This may require a process of frequent reappraisal of diuretic regimen in the face of changes in disease status, concomitant medication, and dietary sodium and fluid intake. Adjustment of diuretic dosing according to fluid overload has been related with more favorable prognosis. ${ }^{10,11}$ In our clinic, furosemide dose is determined during each appointment in respect to the actual volume status (presence of edema, rales, orthopnea, weight control, jugular venous pressure) and overall clinical condition (NYHA class, laboratory test), assuring that the prescribed therapy responds to the individual's demands. The need for dynamic furosemide dose analysis was reaffirmed by the frequent dose readjustments; one-half of patients altered diuretic dose within 1 year after referral, more often toward higher doses. ${ }^{12}$ Despite widespread support for titrated diuretic dose in HF management, prognostic implication of time-varying diuretic dosage has not been thoroughly investigated. Abdel-Qadir et al ${ }^{13}$ suggested that exposure to higher furosemide dose was associated with increased risk of hospital readmission and death; however, the effect of the dynamic dose was controlled for baseline covariates only. In our study, after multiple adjustments for time-varying factors and the risk of death were not significantly different between patients treated with low and high doses.

In a sensitivity analysis, we demonstrated that, in the medium term, the baseline diuretic dose carries a distinct prognostic value, explaining at least in part the strong association reported previously for shorter follow-up. ${ }^{14,15}$ However, in the longer perspective, its importance decreased in favor of the time-varying dose (Appendix S1, Supplementary Table B, model 6).

The study provided detailed information on outpatient diuretic exposure over time and extensive characterization of disease severity and other potential confounders for inclusion in the time-dependent analysis in a relatively large sample of patients.

We did not consider in-hospital and postdischarge diuretic doses as well as possible influence of the patient's adherence to medications on mortality. In our clinic, furosemide is the only loop diuretic prescribed to chronic patients; a possible short course of metolazone translates into a negligible exposure time. The group of 58 excluded patients was not significantly different from the participants in baseline characteristics; therefore, a putative selection bias is expectedly small. The use of medical records of routine care contributes to the pragmatic generalizability of the results; however, findings of this single-center study may not be extrapolated to different settings.

In conclusion, ambulatory HF patients experienced frequent furosemide dose adjustment, usually toward higher doses. Intensity of diuretic treatment seems to reflect the severity of patient's condition, and its chronic use had no independent effect on 5-year survival. Our results confirm that aggressive diuretic therapy may be safe if adequately tailored for congestion status. 


\section{Disclosures}

There are no conflicts of interests.

\section{Acknowledgments}

This study was funded by FEDER through the Operational Programme Competitiveness and Internationalization and national funding from the Foundation for Science and Technology- FCT (Portuguese Ministry of Science, Technology and Higher Education) (FCOMP-01-0124-FEDER011019), under the project "A pharmacoepidemiological approach to the study of prognosis in acute and chronic heart failure" (Ref. FCT PTDC/SAU-ESA/107940/2008) and the Unidade de Investigação em Epidemiologia-Instituto de Saúde Pública da Universidade do Porto (EPIUnit) (POCI01-0145-FEDER-006862; Ref. UID/DTP/04750/2013).

\section{Appendix: Supplementary Data}

Supplementary data related to this article can be found at doi:10.1016/j.cardfail.2017.04.001.

\section{References}

1. Guglin M. Congestion is the driving force behind heart failure. Curr Heart Fail Rep 2012;9:219-27.

2. Faris RF, Flather M, Purcell H, Poole-Wilson PA, Coats AJ. Diuretics for heart failure. Cochrane Database Syst Rev 2012;(2):CD003838.

3. DeVore AD, Hasselblad V, Mentz RJ, O'Connor CM, Armstrong PW, McMurray JJ, et al. Loop diuretic dose adjustments after a hospitalization for heart failure: insights from ASCEND-HF. Eur J Heart Fail 2015; 17:340-6.

4. Writing Committee M, Yancy CW, Jessup M, Bozkurt B, Butler J, Casey DE Jr, et al. 2013 ACCF/AHA guideline for the management of heart failure: a report of the American College of Cardiology Foundation/
American Heart Association Task Force on practice guidelines. Circulation 2013; 128:e240-327.

5. McMurray JJ, Adamopoulos S, Anker SD, Auricchio A, Bohm M, Dickstein K, et al. ESC guidelines for the diagnosis and treatment of acute and chronic heart failure 2012: The Task Force for the Diagnosis and Treatment of Acute and Chronic Heart Failure 2012 of the European Society of Cardiology. Developed in collaboration with the Heart Failure Association (HFA) of the ESC. Eur J Heart Fail 2012;14:803-69.

6. Alibay Y, Schmitt C, Beauchet A, Dubourg O, Alexandre JA, Boileau $\mathrm{C}$, et al. Non-radioimmunometric NT-ProBNP and BNP assays: impact of diluent, age, gender, BMI. Ann Biol Clin (Paris) 2005;63:43-9.

7. Bhavnani SP, Kumar A, Coleman CI, Guertin D, Yarlagadda RK, Clyne $\mathrm{CA}$, et al. The prognostic impact of pre-implantation hyponatremia on morbidity and mortality among patients with left ventricular dysfunction and implantable cardioverter-defibrillators. Europace 2013;16: doi:10.1093/europace/eut211

8. Mielniczuk LM, Tsang SW, Desai AS, Nohria A, Lewis EF, Fang JC, et al. The association between high-dose diuretics and clinical stability in ambulatory chronic heart failure patients. J Card Fail 2008;14:388-93.

9. Damman K, Kjekshus J, Wikstrand J, Cleland JG, Komajda M, Wedel $\mathrm{H}$, et al. Loop diuretics, renal function and clinical outcome in patients with heart failure and reduced ejection fraction. Eur J Heart Fail 2016;18:328-36.

10. Parrinello G, Torres D, Paterna S, Di Pasquale P, Trapanese C, Cardillo $\mathrm{M}$, et al. Early and personalized ambulatory follow-up to tailor furosemide and fluid intake according to congestion in post-discharge heart failure. Intern Emerg Med 2013;8:221-8.

11. Terrovitis J, Kapelios C, Kaldara E, Ntalianis A, Sousonis V, Sfakianaki T, et al. High furosemide doses increase mortality and morbidity in stable chronic heart failure: a prospective, randomized study. J Am Coll Cardiol 2013;61.

12. Piano MR, Prasun MA, Stamos T, Groo V. Flexible diuretic titration in chronic heart failure: where is the evidence? J Card Fail 2011;17:94454.

13. Abdel-Qadir HM, Tu JV, Yun L, Austin PC, Newton GE, Lee DS. Diuretic dose and long-term outcomes in elderly patients with heart failure after hospitalization. Am Heart J 2010;160:264-71.e1.

14. Eshaghian S, Horwich TB, Fonarow GC. Relation of loop diuretic dose to mortality in advanced heart failure. Am J Cardiol 2006;97:1759-64.

15. Martins J, Lourenco P, Araujo JP, Mascarenhas J, Lopes R, Azevedo A, et al. Prognostic implications of diuretic dose in chronic heart failure. J Cardiovasc Pharmacol Ther 2011;16:185-91. 